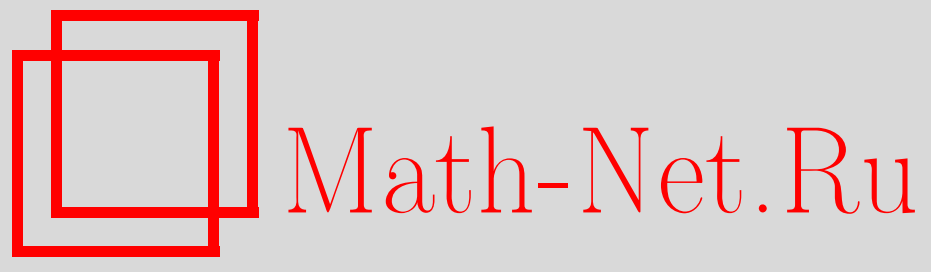

А. В. Алексеевский, С. М. Натанзон, Алгебра чисел Гурвица лоскутных поверхностей, УМН, 2006, том 61, выпуск 4, 185-186

DOI: https://doi.org/10.4213/rm2610

Использование Общероссийского математического портала Math-Net.Ru подразумевает, что вы прочитали и согласны с пользовательским соглашением http://www .mathnet.ru/rus/agreement

Параметры загрузки:

IP : 52.87 .193 .239

26 апреля 2023 г., 16:49:12

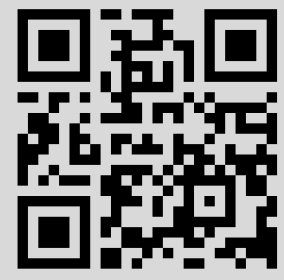




\section{Алгебра чисел Гурвица лоскутных поверхностей}

\section{А. В. Алексеевский, С. М. Натанзон}

1. В настоящей статье мы распространяем теорию чисел Гурвица на клейновы лоскутные поверхности, возникающие в современной математической физике [1]. Клейновой поверхностью называется компактная поверхность, возможно неориентируемая и/или с границей, снабженная атласом локальных карт со значениями в $\{z \in \mathbb{C} \mid \operatorname{Im}(z) \geqslant 0\}$, функции перехода между которыми голоморфны или антиголоморфны [2]. Морфизмами клейновых поверхностей являются отображения, которые голоморфны или антиголоморфны в локальных картах и переводят граничные точки в граничные. Клейновы лоскутные поверхности [1] получаются из конечного множества клейновых поверхностей (лоскутов) склейкой по участкам границы, гомеоморфным отрезкам или контурам. При этом несколько (в частности, более двух) лоскутов могут склеиваться по общему отрезку или контуру. Требуется, чтобы возникающие на границе двух лоскутов вещественные переходные функции были аналитическими во внутренних точках границы. (В отличие от определения [1] наше определение допускает неориентируемые клейновы поверхности.) Морфизмами клейновых лоскутных поверхностей являются отображения, ограничение которых на каждый лоскут является морфизмом клейновых поверхностей. Два таких морфизма $f: \widetilde{\Omega} \rightarrow \Omega$ и ${\underset{\sim}{\Omega^{\prime}}:}_{\widetilde{\Omega}^{\prime}} \rightarrow \Omega$ называются эквивалентными, если существует обратимый морфизм $\varphi: \widetilde{\Omega}^{\prime} \rightarrow \widetilde{\Omega}$ такой, что $f \varphi=f^{\prime}$.

Пусть $f: \widetilde{\Omega} \rightarrow \Omega$ - морфизм клейновой лоскутной поверхности на клейнову поверхность такой, что число прообразов внутренней точки общего положения равно $n$ (т.е. морфизм степени $n$ ). Мы будем считать, что граница $\partial \Omega$ ориентирована и, более того, эта ориентация порождается одной из глобальных ориентаций поверхности $\Omega$ в случае, когда поверхность $\Omega$ ориентируема. Локальным топологическим инвариантом морфизма $f$ в точке $p \in \Omega-\partial \Omega$ назовем таблицу Юнга $\alpha(f, p)$ степени $n$, представляющую собой набор степеней отображения $f$ в точках полного прообраза $f^{-1}(p)=\left\{p^{1}, \ldots, p^{k}\right\}$. Точка $p \in \Omega-\partial \Omega$ называется критическим значением отображения $f$, если число ее прообразов меньше $n$. Свяжем с граничной точкой $q \in \partial \Omega$ множество $f^{-1}(l)$, где $l$ - замыкание множества $\partial D-(\partial D \cap \partial \Omega)$ и $D \subset \Omega$ достаточно малая дисковая окрестность точки $q$. Прообраз $f^{-1}(l)$ отрезка $l$ является двудольным графом с возможно кратными ребрами. Ориентация границы $\partial \Omega$ порождает упорядочение концов отрезка $l$, определяющее упорядочение двух подмножеств (долей) на которые разбиты вершины графа. Класс топологической эквивалентности $\beta(f, q)$ двудольного графа с упорядоченными долями $f^{-1}(l)$ и будет считаться локальным топологическим инвариантом морфизма $f$ в точке $q \in \partial \Omega$. Точка $q$ называется граничным критическим значением, если хотя бы одна из связных компонент графа $\beta(f, q)$ имеет более 2 вершин.

Рассмотрим теперь клейнову поверхность $\Omega$ с множеством (ориентированных, как и выше) граничных контуров $c_{1}, \ldots, c_{s}$. Пусть $p_{1}, \ldots, p_{m} \in \Omega-\partial \Omega-$ множество попарно несовпадающих внутренних точек и $q_{1}^{i}, \ldots, q_{m_{i}}^{i} \in c_{i}$ - множество попарно несовпадающих граничных точек, нумерация которых соответствует ориентации контура $c_{i}$. Число Гурвица клейновых лоскутных поверхностей определяется как $H\left(\Omega,\left\{\alpha_{i}\right\},\left\{\beta_{j}^{i}\right\}\right)=\sum_{f \in \operatorname{Cov}_{n}\left(\Omega,\left\{\alpha_{i}\right\},\left\{\beta_{j}^{i}\right\}\right)} 1 /|\operatorname{Aut}(f)|$, где $\operatorname{Cov}_{n}\left(\Omega,\left\{\alpha_{i}\right\},\left\{\beta_{j}^{i}\right\}\right)-$ множество классов эквивалентности морфизмов $f: \widetilde{\Omega} \rightarrow \Omega$, все критические значения которых принадлежат множеству $\left(\bigcup_{i} p_{i}\right) \cup\left(\bigcup_{i, j} q_{j}^{i}\right)$ и $\alpha\left(f, p_{i}\right)=\alpha_{i}, \beta\left(f, q_{j}^{i}\right)=\beta_{j}^{i}$ для всех $i, j$. Через $|\operatorname{Aut}(f)|$ обозначен порядок группы автогомеоморфизмов поверхности $\widetilde{\Omega}$, сохраняющих отображение $f$. Наше определение переходит в определение чисел Гурвица комплексных [3] (соответственно вещественных [4]) алгебраических кри-

Работа выполнена при поддержке грантов РФФИ-04-01-00762, NWO 047.011.2004.026 (РФФИ 05-02-89000-HВО-а), НШ-4719.2006.1, CRDF RM1-2543-MO-03. 
вых, если все рассматриваемые поверхности являются римановыми (соответственно клейновыми).

2. Числа Гурвица клейновых лоскутных поверхностей вычисляются с помощью фробениусовых алгебр, описанных ниже. Рассмотрим группу перестановок $S_{E}$ множества $E$ из $n$ элементов. Обозначим через $\Sigma=\Sigma(E)$ множество всех разбиений $E$ на подмножества и через $\Sigma_{m}=\Sigma_{m}(E)$ - множество всех упорядоченных наборов $\left(\sigma_{1}, \ldots, \sigma_{m}\right)$ разбиений $\sigma_{i} \in \Sigma$. Группа $S_{E}$ действует на $\Sigma_{m}$ по правилу $s\left(\sigma_{1}, \ldots, \sigma_{m}\right)=\left(s \sigma_{1}, \ldots, s \sigma_{m}\right)$. Обозначим через $\mathscr{A}_{m}=\mathscr{A}_{m}(E)$ множество орбит этого действия. Множество $\mathscr{A}_{1}=\left\{a_{i}\right\}$ естественно отождествляется с множеством таблиц Юнга степени $n$, с множеством классов сопряженности элементов группы $S_{E}$ и со стандартным базисом центра $A$ групповой алгебры над полем $\mathbb{Q}$ группы $S_{E} \cong S_{n}$. Обозначим через $l^{A} \in A^{*}$ линейный функционал, принимающий значение 1 на тождественной перестановке и 0 на остальных перестановках.

Множество $\mathscr{A}_{2}=\left\{b_{i}\right\}$ естественно отождествляется с множеством классов топологической эквивалентности двудольных графов с $n$ ребрами и упорядоченными долями (пара разбиений - это разбиения ребер графа на группы, индуцированные двумя группами вершин). Рассмотрим векторное пространство $B$ над $\mathbb{Q}$ с базисом $\mathscr{A}_{2}$. Обозначим через $V=V_{E}$ векторное над $\mathbb{Q}$ с базисом $\Sigma$ и через $M=M_{E}$ алгебру эндоморфизмов $V$. Действие $S_{E}$ на $\Sigma$ порождает гомоморфизм $\rho: S_{E} \rightarrow M$. Сопоставим паре разбиений $\left(\sigma_{i}, \sigma_{j}\right)$ эндоморфизм $M_{\sigma_{i}, \sigma_{j}}$, такой, что $M_{\sigma_{i}, \sigma_{j}} \sigma_{r}=\delta_{\sigma_{i}, \sigma_{r}} \sigma_{j}$. Равенство $\psi(\beta)=\sum_{\left(\sigma_{i}, \sigma_{j}\right) \in \beta} M_{\sigma_{i}, \sigma_{j}}$ порождает мономорфизм $\psi: B \rightarrow M$, индуцирующий на $B$ структуру алгебры.

Образ $\psi(B)$ состоит из всех эндоморфизмов, коммутирующих с $\rho\left(S_{E}\right)$.

Рассмотрим линейный функционал $l^{B}: B \rightarrow \mathbb{Q}$, сопоставляющий элементу $b \in B$ след матрицы $\psi(b)$.

Сопоставим перестановке $x \in S_{E}$ класс $a(x) \in \mathscr{A}_{1}$ разбиения множества $E$ на орбиты элемента $x^{2}$. Положим $U=\sum_{a \in \mathscr{A}_{1}} \frac{d(a) a}{n !} \in A$, где $d(a)$ - число элементов $x \in S_{E}$ таких что $a(x)=a$. Обозначим через $\left\{F_{A}^{i j}\right\}$ и $\left\{F_{B}^{i j}\right\}$ - матрицы, обратные к $\left\{F_{i j}^{A}\right\}=\left\{l^{A}\left(a_{i} a_{j}\right)\right\}$ и $\left\{F_{i j}^{B}\right\}=\left\{l^{B}\left(b_{i} b_{j}\right)\right\}$. Положим $K_{A}=F_{A}^{i j} a_{i} a_{j}$. Зададим оператор $V_{K_{B}}: B \rightarrow B$ условием $V_{K_{B}}(b)=F_{B}^{i j} b_{i} b b_{j}$. Зададим линейный оператор $\phi: A \rightarrow B$ условием $\phi\left(a_{k}\right)=R_{k, i} F_{B}^{i j} b_{j}$, где $R_{k, i}=\frac{1}{n !} \sum_{x \in a_{k},\left(\sigma_{1}, \sigma_{2}\right) \in b_{i}} \delta_{x\left(\sigma_{1}\right), \sigma_{2}}$.

Теорема. Числа Гурвица равны $H\left(\Omega,\left\{\alpha_{i}\right\},\left\{\beta_{j}^{i}\right\}\right)=l^{B}\left(\phi\left(\alpha_{1} \cdots \alpha_{m} L^{g}\right)\left(\beta_{1}^{1} \cdots \beta_{m_{1}}^{1}\right) \times\right.$ $\left.V_{K_{B}}\left(\beta_{1}^{2} \cdots \beta_{m_{2}}^{2}\right) \cdots V_{K_{B}}\left(\beta_{1}^{s} \cdots \beta_{m_{s}}^{s}\right)\right)$, где $g-$ род поверхности $\Omega$ в смысле [4] $u L=K_{A}$ (соответственно $L=U^{2}$ ) для ориентируемой (соответственно неориентируемой) поверхности $\Omega$.

Авторы благодарят IHES, где делалась часть работы над статьей.

\section{Список литературы}

[1] L. Rozansky, "Topological A-models on seamed Riemann surfaces", arXiv: hep-th/0305205. [2] С. М. Натанзон, УМH, 45:6 (1990), 47-90. [3] A. Hurwitz, Math. Ann., 39 (1891), 1-60. [4] A. Alexeevski, S. Natanzon, "Non-commutative extensions of two-dimensional topological field theories and Hurwitz numbers for real algebraic curves", arXiv: math.GT/0202164.

А. В. Алексеевский (А. V. Alekseevskii)

Московский государственный университет им. М. В. Ломоносова

E-mail: aba@genebee.msu.ru

\section{C. М. Натанзон (S. M. Natanzon)}

Московский государственный университет им. М. В. Ломоносова,

Институт теоретической и экспериментальной физики,

Независимый московский университет

E-mail: natanzon@mccme.ru
Представлено С. П. Новиковым Принято редколлегией 18.01.2006 